\title{
Situation de référence de la phytodiversité et la productivité herbacée d'un dispositif de suivi du feu de brousse au Niger
}

\author{
Maman Maârouhi INOUSSA ${ }^{1 *}$, Abdoulaye DIOUF ${ }^{2}$, Yacoubou BAKASSO ${ }^{1}$, \\ Boubé MOROU ${ }^{2}$, Maïnassara ZAMAN-ALLAH ${ }^{2}$, Ali MAHAMANE ${ }^{1,2}$ \\ et Mahamane SAADOU ${ }^{1,2}$ \\ ${ }^{1}$ Université Abdou Moumouni de Niamey / Faculté des Sciences et Techniques, Département de Biologie, \\ Laboratoire Garba Mounkaila, BP : 10662 Niamey, Niger. \\ ${ }^{2}$ Université de Maradi / Faculté d'Agronomie et des Sciences de l'Environnement, \\ Département des Sciences du Sol et de Télédétection, Maradi, Niger. \\ ${ }^{3}$ Université de Maradi / Faculté des Sciences et Techniques, Département de Biologie, Maradi, Niger. \\ *Auteur correspondant ; E-mail : maman_maarouhi@yahoo.fr
}

\section{RESUME}

Cette étude entre dans le cadre de la mise en place d'un dispositif de suivi des impacts des feux dans la Réserve Totale de Faune de Tamou contiguë au Parc Régional du W. Pour cela, la phytomasse a été prélevée par la méthode de la récolte intégrale et les relevés phytosociologiques ont été faits suivant l'approche BraunBlanquet. L'Analyse Factorielle des Correspondances (AFC) a été appliquée à une matrice relevés X espèces pour l'individualisation des groupements à l'aide du logiciel CANOCO 5.1. Pour l'interprétation écologique des résultats, une Analyse Canonique des Correspondances (ACC) a été réalisée sur la matrice relevés X variables environnementales. Au total, 107 espèces ont été recensées dont 37 ligneuses et 70 herbacées, reparties dans 80 genres et 39 familles. L'AFC a mis en évidence 7 groupements suivant un gradient nord-sud. Quatre (4) de ces groupements sont des faciès de savanes arborées +/- arbustives, localisées dans la partie sud de la station expérimentale et les trois (3) autres, situés au nord sont des savanes herbeuses. Par ailleurs, l'analyse de la diversité alpha au sein de ces différents faciès végétaux a fait ressortir un gradient d'augmentation de la phytodiversité selon le type de faciès. Quant à la productivité herbacée, elle varie dans le sens inverse.

(C) 2014 International Formulae Group. All rights reserved.

Mots clés: Savane ; productivité herbacée, phytodiversité, feux de brousse, Réserve Totale de Faune de Tamou, Niger.

\section{INTRODUCTION}

Le feu est un élément fondamental et naturel $\mathrm{du}$ fonctionnement de nombreux écosystèmes forestiers. Pendant des millénaires, les êtres humains ont utilisé le feu comme outil de gestion des terres. C'est l'une des forces naturelles qui a influencé les communautés végétales au fil des siècles et, comme processus naturel, il exerce une importante fonction dans le maintien du fonctionnement de certains écosystèmes (Dennis et al., 2001). En effet, les écosystèmes de la zone des savanes sont caractérisés par une forte concurrence entre 
les arbres et les herbes. L'équilibre entre ces deux composantes dépend largement des effets interactifs indirects entre l'herbivorie et le feu (Van Langevelde et al., 2003). Généralement, les stations qui sont fortement pâturées sont moins parcourues par le feu. L'action des feux a été analysée dans divers écosystèmes du monde. David et al. (2001) retracent l'impact des feux de brousse en faisant l'historique de la forêt. Le passage du feu entraîne une relative diminution de la couverture des ligneux (Sankaran et al., 2005). Dans certains cas, il entraînerait la disparition de certaines espèces pyrophobes (Diouf et al., 2012). Des travaux plus récents montrent qu'en zone soudanienne, la dynamique de la végétation ligneuse est en grande partie tributaire des feux (Diouf et al. 2012 a et Diouf, 2012). Selon ces auteurs, les impacts des feux de végétation varient dans l'espace et dans le temps selon les conditions écologiques. Ainsi, même sur des stations favorables, les feux répétés entraînent la disparition des espèces ligneuses qui n'ont pas développé des adaptations morphologiques ou anatomiques. Souvent, c'est la phénologie des espèces qui se trouve perturbé avec le passage du feu (Adam, 1977). Les feux jouent ainsi un rôle sur la productivité des biotopes (Peter et al., 2001). Par ailleurs, les feux de végétation jouent un rôle dans l'émission des gaz lors des brûlis (Mbow, 2009). Pourtant, les gestionnaires du parc $\mathrm{W}$ utilisent les feux précoces pour lutter contre les feux tardifs plus dévastateurs de la végétation ligneuse et obtenir des repousses de graminées pérennes bien appréciées par les herbivores. Les données existantes sur les feux de végétation ont porté sur les impacts de ceux-ci sur la structure de la strate ligneuse (Diouf, 2012). Or, la composante herbacée joue un rôle non négligeable dans la propagation de feu de brousse et les herbes pourraient être plus fragiles que les ligneux. Par ailleurs, dans le Parc W, il manque des données sur la composition et le taux des gaz émis dans l'atmosphère au cours de la combustion. Alors, il s'avère urgent de mener des études sur les régimes de feu et l'émission des gaz qu'ils engendrent à travers des sites expérimentaux. Le but étant d'obtenir des données scientifiques sur les différents régimes de feu et le suivi à long terme de leurs impacts sur la dynamique de la végétation d'une part et l'émission des gaz dans l'atmosphère d'autre part. Le présent article a pour objectif principal de caractériser la végétation d'un site expérimental installé dans la Reserve Totale de Faune de Tamou en périphérie du Parc National du W. Ce qui permettra d'assoir un dispositif de suivi des effets de feu sur la végétation et l'atmosphère. Il s'agit en fait de collecter des informations qui seront considérées comme données au temps $\mathrm{t}=0$ avant la mise à feux. Les objectifs spécifiques de l'étude sont : (1) évaluer la phytodiversité du site ; (2) caractériser les différents faciès de savane en présence ; (3) estimer la productivité de la strate herbacée et (4) analyser la variation de la productivité herbacée en fonction de la diversité alpha suivant les faciès de savane identifiés.

\section{MATERIEL ET METHODES Zone d'étude}

La Réserve Totale de Faune de Tamou (R.T.F.T), créée le 08 août 1962, est située dans la partie sud-ouest du Niger et fait partie, sur le plan administratif, de la commune rurale de Tamou, Département de Say (Région de Tillabéri). Elle est limitée par la rivière Diamangou au nord, le territoire burkinabé à l'ouest, le Parc National du W au sud et à l'est par la zone "AYINOMA" issue de la réduction de la superficie de la R.T.F.T (de 140.000 à 76000 ha) par décret $\mathrm{N}^{\circ} 76-141 /$ MDR du 12 Août 1976. Elle est comprise entre les latitudes $12^{\circ} 28^{\prime}$ et $12^{\circ} 50^{\prime}$ nord et les longitudes $2^{\circ} 06^{\prime}$ et $2^{\circ} 24^{\prime}$ est (Figure 1 ).

Le climat est de type tropical soudanien (Lamarque, 2004). Le site étudié se localise sur le bas-versant du plateau occidental dont le bas-fond correspond à la rivière Tapoa (Couteron et Kokou, 1992). La zone est juridiquement inhabitée, mais elle est soumise aux pressions de la population riveraine du Canton de Tamou (114518 habitants). Cette zone a enregistré le plus 
grand flux de migrants dans ces dernières décennies (Amadou, 2006). Ce mouvement démographique est dû à l'existence des meilleures conditions agro-écologiques et la disponibilité des terres couplée à la souplesse du régime foncier et au manque des ressources dans les régions d'origine des migrants. La population est constituée d'une mosaïque d'ethnies aux traditions culturelles et religieuses diverses: Baribas, Peuls, Gandos, Dendis, Haoussas, Mokoles, Gourmantchés et Foulangani.

\section{Méthodes}

\section{Choix du site}

Plusieurs travaux sur la végétation du Parc Régional du W sont conduits par des chercheurs du Laboratoire GARBA Mounkaïla de Biologie responsable du dispositif étudie (Mahamane, 2005; Barbier, 2006 ; Inoussa, 2011; Diouf, 2012). Ces travaux ont analysé la structure et la dynamique de la végétation et un dispositif de suivi de la phénologie des essences ligneuses est défini (Mahamane, 2005). Suite à ces travaux, le Projet ECOPAS a sollicité un dispositif de suivi des feux d'aménagement à travers 8 stations réparties en fonction des principaux types de végétation (Diouf, 2007). Le choix initial des sites a été fait non seulement sur la base de critères physionomiques et environnementaux (géomorphologie ; topographie ; type de substrat ; la profondeur du sol et le passage du feu) mais également en fonction de l'accessibilité des sites en toutes saisons. Le travail porte sur une station qui est située dans la Réserve Totale de Faune de Tamou sousjacente au Parc National du W du Niger.

\section{Dispositif expérimental}

Sur le site, 16 parcelles de $50 \mathrm{~m}$ x $50 \mathrm{~m}$ (soit 1/4 hectare chacune) ont été délimitées. L'ensemble de ces parcelles constitue le dispositif expérimental aux dimensions $250 \mathrm{x}$ $250 \mathrm{~m}$. Chaque parcelle a été ensuite subdivisée en 25 placeaux de $100 \mathrm{~m}^{2}$ délimités chacun par des bornes métalliques à chaque angle afin d'alléger l'inventaire floristique
(Figure 2). Au moment de l'application des feux, tout autour du dispositif et des parcelles constitutives, des pare-feu de $10 \mathrm{~m}$ de large seront réalisés afin de permettre un bon contrôle des feux pendant le traitement. Quatre dates de mise à feu (du début de dessiccation de l'herbe jusqu'au dessèchement complet de celle-ci) seront expérimentées. Les trois premières dates de mise à feu sont suivies avec un intervalle de 10 jours. Puis à la quatrième date, soit un mois plus tard, il sera appliqué un feu tardif. Quatre (4) parcelles, selon l'axe Nord-Sud par dispositif, recevront l'un des quatre traitements de feu $\left(« 1^{\mathrm{er}}\right.$ feu précoce $», \ll 2^{\mathrm{ème}}$ feu précoce», «feu tardif» et «pas de feu »).

\section{Récoltes des données \\ Relevés phytoécologiques}

Les placeaux de relevés ont été placés suivant la diagonale de chaque placette du dispositif (Figure 2). Les relevés phytosociologiques ont été effectués suivant l'approche sigmatiste de Braun-Blanquet (Guinochet, 1973). Au total, 80 placeaux ont été relevés. Dans chaque placeau, les espèces présentes ont été recensées, chacune affectée d'un coefficient d'abondance-dominance suivant l'échelle de Braun Blanquet. L'abondance-dominance exprime le nombre d'individus d'une même espèce et le degré de leur recouvrement. Les relevés ont été effectués en fin de la saison des pluies (Septembre-Octobre), période pendant laquelle la végétation est à l'optimum de son développement (Saadou, 1990). Au cours des relevés phytosociologiques, des informations stationnaires ont été également notées. Il s'agit: des états de surface selon la typologie de Casenave et Valentin (1990) et la texture du sol.

\section{Prélèvement de la phytomasse herbacée}

La mesure a concerné la strate herbacée épigée accessible aux herbivores sauvages et au feu. Il existe plusieurs méthodes de mesure de la phytomasse herbacée et, Djitéye (1988) en a fait la synthèse. C'est ainsi qu'on peut noter: les 
méthodes non destructives, les méthodes semi-destructives et les méthodes destructives. Ce sont ces dernières qui sont appliquées dans la présente étude. Il s'agit d'une technique classique de la récolte intégrale (méthode directe) permettant la pesée de la biomasse prélevée. La méthode est simple même si elle ne permet pas le suivi de la végétation, car elle modifie sa structure.

La mesure a été réalisée à l'aide de carrés métalliques de $1 \mathrm{~m}^{2}$ (Baina, 2000). Dans chaque placeau ayant fait l'objet des relevés phytoécologiques, un carré de phytomasse herbacée a été prélevé au milieu du placeau. La récolte s'est déroulée de la façon suivante :

- après avoir posé le carré, les herbes dont les racines sont placées en dehors du carré sont bien séparées de celles dont les racines sont à l'intérieur ;

- la coupe des herbes s'est faite à l'aide d'un sécateur au ras du sol pour les annuelles, à $10 \mathrm{~cm}$ de hauteur pour les vivaces (comme Andropogon gayanus) ;

- toutes les herbes coupées ont été soigneusement récoltées et mises dans un sachet plastique dont on connaît le poids ; - après séchage, le poids sec a été mesuré pour l'estimation de la phytomasse.

\section{Analyse des données}

L'ordination des relevés a été abordée par les techniques d'analyses multivariées. Il s'agit ici de l'Analyse Factorielle des Correspondances (AFC). Cette méthode a été réalisée à l'aide du logiciel CANOCO (Canocal Community Ordination) for Windows, version 3.1 (Ter Braak et Smilauer, 1998). Ainsi, une matrice de 80 relevés et 107 espèces a été soumise à l'AFC. L'analyse est basée sur des tableaux floristiques et permet de réduire la variabilité floristique entre relevés à un nombre réduit d'axes factoriels. L'application de l'Analyse Canonique des Correspondances (ACC) a permis d'évaluer la proportion de la variabilité floristique pouvant être expliquée par les variables environnementales. Ainsi, la matrice de 80 relevés croisée aux variables environnementales a été soumise à l'ACC. Les modalités des différents descripteurs du milieu physique ont été testées pour cibler celles qui déterminent des gradients. La nomenclature des groupements identifiés a été effectuée sur la base de la dominance des espèces (Fournier, 2000). La nomenclature des espèces suit celle de Hutchinson et Dalziel (1954-1972) et Lebrun et Storck (1991-1997).

Pour l'appréciation de la diversité alpha au sein des différents groupements, il a été utilisé l'indice de Shannon et Weaver (1949). Cet indice évalue le poids de l'espèce dans l'occupation du sol et varie en fonction du nombre d'espèces présentes. Il est d'autant plus élevé qu'un grand nombre d'espèces participe au recouvrement. Il s'exprime en bits par individu. D’une manière générale, sa valeur varie de 0 à 5 bits.

La valeur 0 correspond à une diversité faible et les valeurs élevées indiquent un nombre élevé d'espèces qui participent au recouvrement.

La formule utilisée est :

$$
H=-\sum p_{i} \log _{2} p_{i}
$$

Avec $P_{i}$ la proportion relative $\mathrm{du}$ recouvrement moyen de l'espèce $i$ dans le groupement (comprise entre 0 et 1 ).

$\log _{2}:$ logarithme à base 2 .

La productivité : c'est la quantité de matière sèche exprimée en t/ha. Son évaluation a été faite groupement par groupement après le calcul de la phytomasse moyenne exprimée en g.ms $/ \mathrm{m}^{2}$ avec l'écart type.

Par la suite, une analyse de variance a été réalisée pour comparer les productivités et les valeurs de l'indice de Shannon-Weaver des différents groupements végétaux à l'aide du logiciel SPSS 11.5. 


\section{RESULTATS ET DISCUSSION Individualisation des groupements}

Les relevés effectués sont au nombre de 80 totalisant 107 espèces dont 37 ligneuses et 70 herbacées réparties dans 80 genres et 39 familles. Le Tableau 1 donne le résumé des résultats de l'Analyse Factorielle des Correspondances (AFC) réalisée sur les données floristiques. D'après ce tableau, les 4 premiers axes factoriels expliquent $3,02 \%$ de la variance totale. Cette faible valeur signifie que le maximum de variabilité se situe entre les relevés et que la part de variabilité environnementale est faible, étant entendu que l'ensemble des relevés a été réalisé sur un même site. La partition des relevés en faciès de savanes est donnée à la Figure 3. Celle-ci illustre l'individualisation de 80 relevés en sept (7) types de savanes. Tous les faciès mis en évidence sont situés dans la partie positive des axes 1 et 2 . L'axe 1 contribue à $34,3 \%$ dans l'ordination des relevés dans le plan factoriel, tandis que l'axe 2 n'explique que 13,5\%. Par rapport à l'axe 1, se dégagent du côté positif les savanes à Loudetia phragmitoides (G7) et à Loudetia togoensis (G6). Le groupement G7 est constitué par des relevés réalisés sur des sols à texture fine alors que les relevés du G6 sont caractérisés par des sols à texture grossière. Vers l'origine de cet axe s'individualise le groupement à Anogeissus leiocarpa (G2) constitué par des relevés sur sol limono-argileux avec des recouvrements notables pour la litière. L'axe 1 correspond donc à un gradient de texture du sol. Suivant l'axe 2 s'individualisent : du côté positif de l'axe les relevés du groupement à Lannea microcarpa $(\mathrm{G} 1)$ sur sol à forte charge graveleuse avec des blocs de grès et vers l'origine de l'axe les relevés du groupement à Combretum glutinosum (G3) sur sol encroûté en surface. Cet axe correspond donc à un gradient des états de surface.

Les groupements 4 et 5 sont identifiés à partir de l'axe 3 qui oppose les groupements à Acacia macrostachya sur sol gravillonnaire et celui à Andropogon gayanus sur sol limono-argileux dans des microdépressions. Cet axe détermine alors un gradient d'humidité.

\section{Facteurs déterminants de la dynamique des groupements}

Il ressort à l'Analyse Canonique des Correspondances (ACC) que seules les modalités de la texture du sol permettent une discrimination pertinente. Pour ce faire, seules celles-ci ont été utilisées. Le résumé des résultats de l'Analyse Canonique des Correspondances (ACC) est consigné dans le Tableau 2. Cette ACC donne au total 0,272 des valeurs propres. La texture du sol explique à $9 \%$ la variance des espèces avec une inertie totale de 3,020.

La comparaison avec le tableau précédent (Tableau 1) fait ressortir une corrélation des variables écologiques avec les axes factoriels. L'ACC conduit donc réellement à mieux déterminer le rôle des facteurs écologiques dans la distribution des communautés floristiques. Généralement, elle ne provoque pas de changements majeurs des axes de l'AFC originale (Figure 4).

La Figure 4 représente la carte factorielle des 80 relevés associés aux cinq (5) modalités de la texture du sol. Ces résultats montrent qu'il existe une relation entre les axes canoniques et la texture du sol. On rappelle que sur la carte factorielle de l'ACC les variables environnementales sont représentées par des flèches pointées vers la direction où leurs valeurs augmentent. Ainsi, les variables qui ont de longues flèches sont plus corrélées à la structure que montrent les données dans le diagramme. Par conséquent, sur cette figure, les textures argileuse, argilosableuse et argilo-limoneuse sont les plus corrélées avec les axes canoniques.

L'axe 1 est un gradient de diminution des textures argileuse, argilo-limoneuse et gravillonnaire et contribue à $10,6 \%$ dans la structuration des relevés tandis que l'axe 2 est 
un gradient de l'accroissement de la texture argilo-sableuse. Il explique la distribution des relevés à seulement $3,7 \%$.

\section{Flore, formes biologiques et types phytogéographiques}

L'analyse de la liste floristique globale fait ressortir une dominance des Fabaceae (12 espèces, $11,2 \%)$ et des Poaceae ( 9 espèces, $8,4 \%)$. Ces résultats confirment ceux obtenus par Saadou (1990) et Mahamane (2005) dans cette même dition, et avec des superficies de relevés beaucoup plus importantes. Il ressort de l'analyse du comportement adaptatif des espèces que les thérophytes dominent au niveau de tous les groupements dans les spectres brut et pondéré. Ceci pourrait s'expliquer par les conditions stationnelles caractérisées par un passage fréquent des feux d'aménagement. Selon Houinato et al., (2001), les feux tardifs favorisent l'apparition de nouvelles thérophytes et provoquent la régression des phanérophytes. Les groupements de savane herbeuse (G6 et G7) se caractérisent par la dominance des thérophytes dans le spectre pondéré à plus de $95 \%$. Il s'agit des parcelles qui se répartissent au côté nord de la station sur le bowal présentant une particularité géomorphologique et édaphique localement (Figures $5 \mathrm{~A}$ et $5 \mathrm{~B}$ ). Des résultats similaires sont obtenus dans les formations végétales sur sol à bowalisé au Bénin (Padonou et al., 2013). Du point de vue phytogéographique, les espèces soudanozambésiennes et guinéo-congolaises-soudanozambésiennes dominent dans tous les groupements au niveau des spectres bruts et pondérés. Aussi, on note une absence ou une présence à faible proportion des espèces soudano-zambéziennes-saharo-indiennes-

méditerranéennes. Ces deux constatations montrent qu'on est dans une zone humide (Figure 6A et 6B). Ceci s'explique par le fait que ce site se localise dans une zone plus ou moins bien arrosée du pays $(700 \mathrm{~mm}$ de pluviométrie moyenne). D'ailleurs, il se situe dans le compartiment Nord soudanien A1 pour lequel Saadou (1990) a eu des résultats similaires.

\section{Diversité et productivité}

Lorsqu'on tient compte du seuil de variation de l'indice de Shannon-Weaver (0 à 5 ), on peut dire que tous les groupements sont relativement diversifiés car les valeurs calculées sont comprises entre 2 et 3,4 bits (Figure 7). Cependant, les groupements de savane arborée plus ou moins arbustive ou arbustive (G1, G2, G3 et G4) comportent les valeurs les plus élevées comparativement aux groupements de savane herbeuse (G5, G6 et G7). Dans ces derniers, on note un effet de dominance par quelques graminées annuelles (Hyparrhenia sulcata, Loudetia togoensis, L. Phragmitoides, etc.), or l'indice de ShannonWeaver est sensible à la dominance, autrement dit, plus il y a dominance, plus sa valeur est faible. En revanche, dans les groupements de savane arborée à arbustive, la multiplication des strates conditionne aussi celle de microhabitats (synusies) qui favorisent l'installation de plusieurs niches écologiques. Par conséquent, la richesse spécifique est importante avec un nombre d'espèces assez appréciable qui participe au recouvrement. Ces résultats sont en accord avec les conclusions de Mahamane (2005) sur la végétation de la terre ferme du Parc Régional W.

La Figure 7, illustre également la variation de la productivité de la strate herbacée selon le type du groupement. On remarque l'augmentation de la productivité de la savane arborée à arbustive dans la partie sud du site à la savane herbeuse dans la partie nord. La valeur la plus faible est de 3,1 tonnes.ms/ha et la plus élevée est de 7,77 tonnes $\mathrm{ms} / \mathrm{ha}$ avec une moyenne de 5,52 tonnes.ms/ha. Ainsi, la productivité peut aussi être utilisée pour caractériser la végétation (Ramade, 1994). La confrontation des valeurs de l'indice de Shannon et de la productivité au 
niveau des différents groupements végétaux, fait ressortir que ceux-ci varient dans le sens opposé (Figure 7). En effet, la productivité est inversement corrélée avec la diversité alpha de façon significative (Tableau 3). Malheureusement, l'application des feux d'aménagement pourrait avoir des impacts sur la dynamique de la végétation sachant que certains groupements sont caractérisés par des espèces pyrophytes tandis que d'autres ont pour caractéristiques des espèces pyrophobes sensibles au feu. Beaucoup de travaux ont abordé cet aspect sans toutefois que les questions soient décantées car les impacts des feux sur la végétation dépendent non seulement du type de faciès, mais aussi des interactions entre les feux et les autres facteurs environnementaux.

Tableau 1: Valeurs propres, longueur des gradients et variance expliquée par les quatre premiers axes de la DCA.

\begin{tabular}{lccccc}
\hline Axes & $\mathbf{1}$ & $\mathbf{2}$ & $\mathbf{3}$ & $\mathbf{4}$ & Inertie totale \\
\hline Valeur propre & 0,343 & 0,131 & 0,100 & 0,086 & 3,020 \\
Longueur des gradients & 2,619 & 1,716 & 1,802 & 1,691 & \\
Pourcentage cumulatif de variance & 11,4 & 15,7 & 19, & 21,8 & \\
expliquée & & & & & \\
\hline
\end{tabular}

Tableau 2 : Résultats de l'ACC après 499 permutations.

\begin{tabular}{lccccc}
\hline Axes & $\mathbf{1}$ & $\mathbf{2}$ & $\mathbf{3}$ & $\mathbf{4}$ & Inertie totale \\
\hline Valeur propres & 0,123 & 0,079 & 0,045 & 0,026 & 3,020 \\
Corrélation Espèces-environnement & 0,705 & 0,817 & 0,727 & 0,665 & \\
Pourcentage cumulé de la variance & & & & & \\
$\quad$ des espèces & 4,1 & 6,7 & 8,2 & 9,0 & \\
$\quad$ de relation espèces-environnement & 45,0 & 73,9 & 90,6 & 100,0 & \\
Somme des valeurs propres & & & & 0,272 & \\
\hline
\end{tabular}

Tableau 3 : Analyse des variances entre les valeurs de l'indice de Shannon et la productivité des différents groupements végétaux.

\begin{tabular}{llcc}
\hline & & Corrélations & \\
\hline \multirow{3}{*}{ Indice } & & Indice & Productivité \\
& Corrélation Pearson & 1 & $0,947^{* *}$ \\
& Sig. (bilatérale) &, & 0,001 \\
& $\mathrm{~N}$ & 7 & 7 \\
\hline \multirow{3}{*}{ Productivité } & Corrélation Pearson & $0,947^{* *}$ & 1 \\
& Sig. (bilatérale) & 0,001 & 7 \\
& $\mathrm{~N}$ & 7 & 7 \\
\hline
\end{tabular}

** La corrélation est significative au niveau 0,01 (bilatéral). 


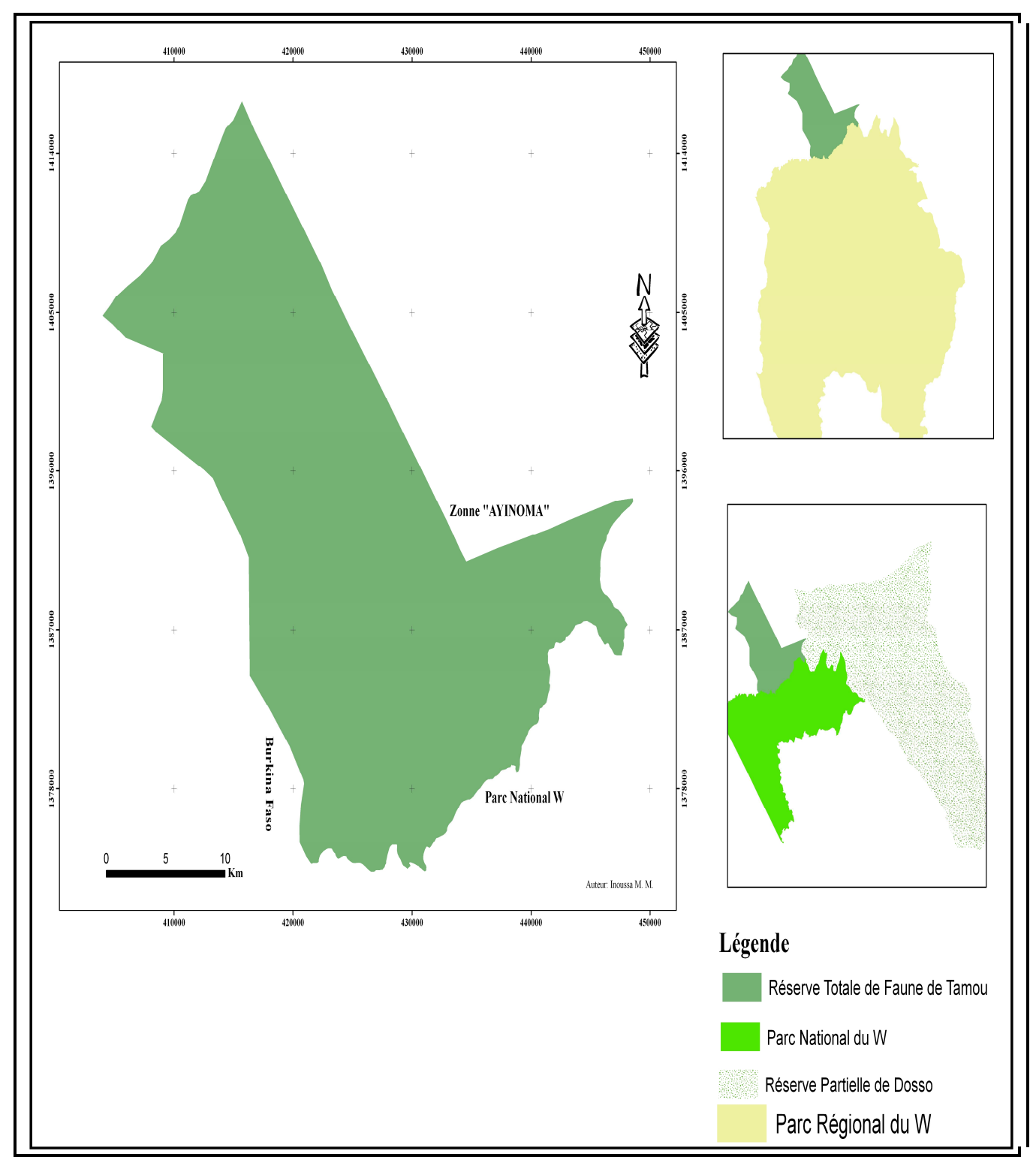

Figure 1: Situation géographique de la Reserve Totale de Faune de Tamou dans le Parc Régional du W du Niger. 


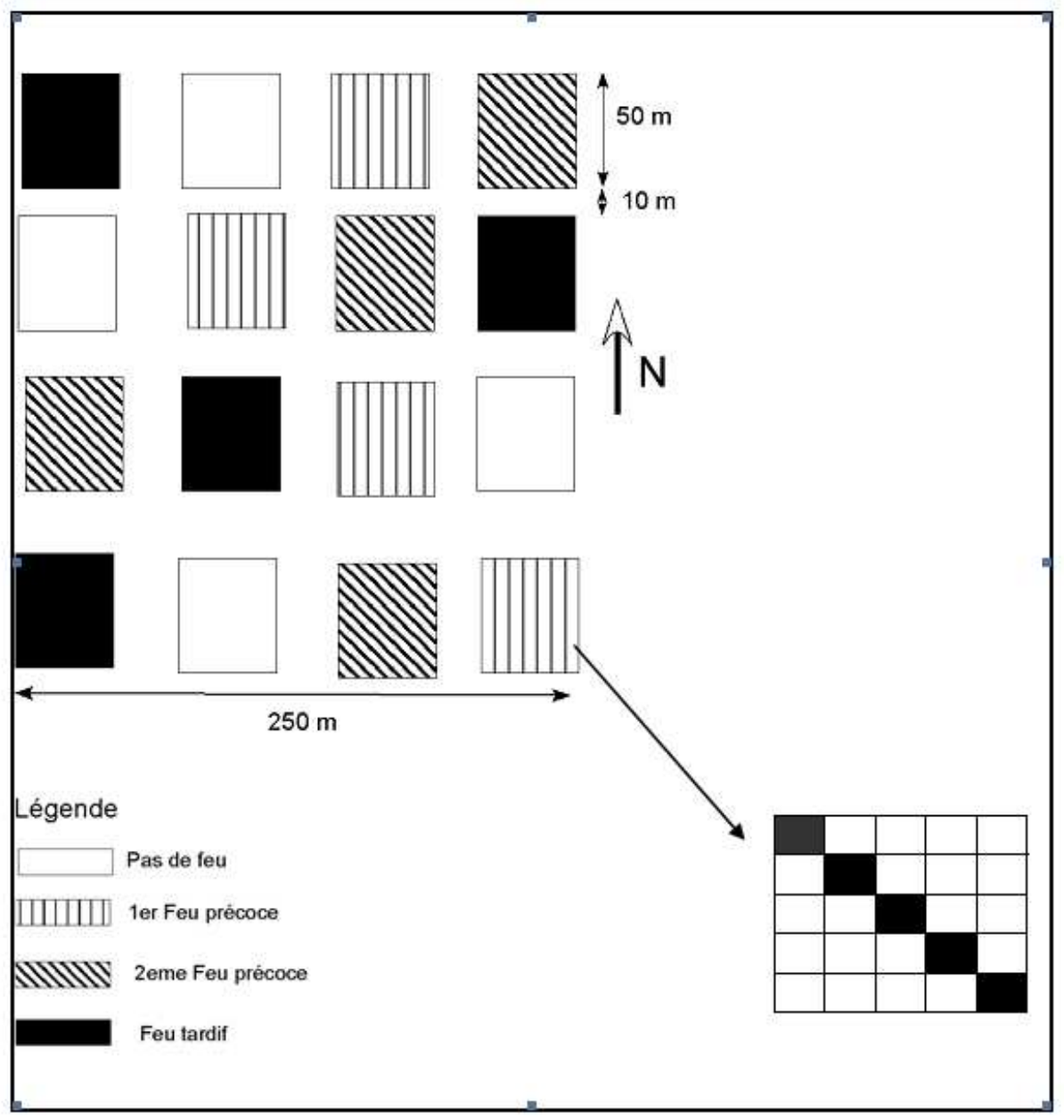

Figure 2: Schéma du dispositif expérimental.

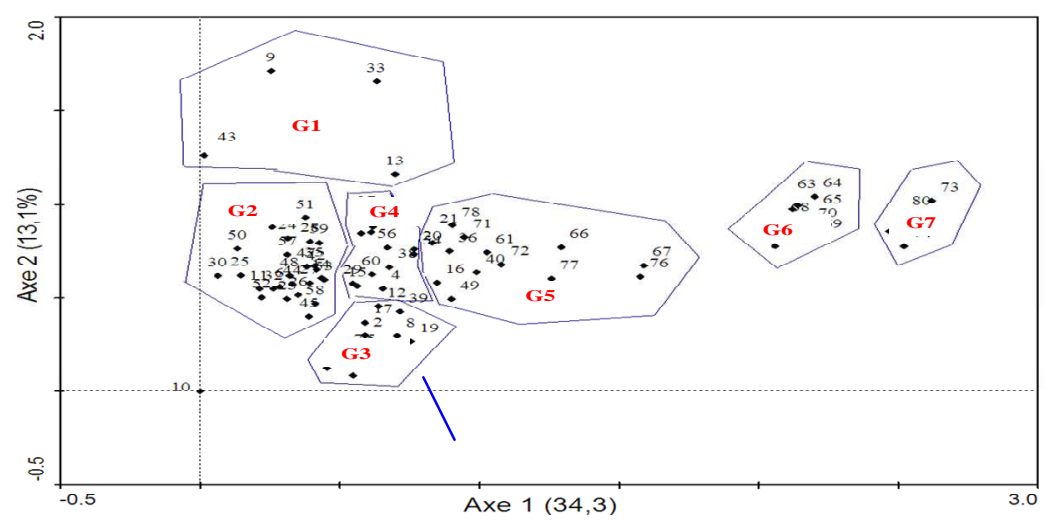

Figure 3 : Diagramme de l'ordination des placeaux dans le plan factoriel des axes 1 et 2 de la DCA. 
Axe $2(3,7 \%)$

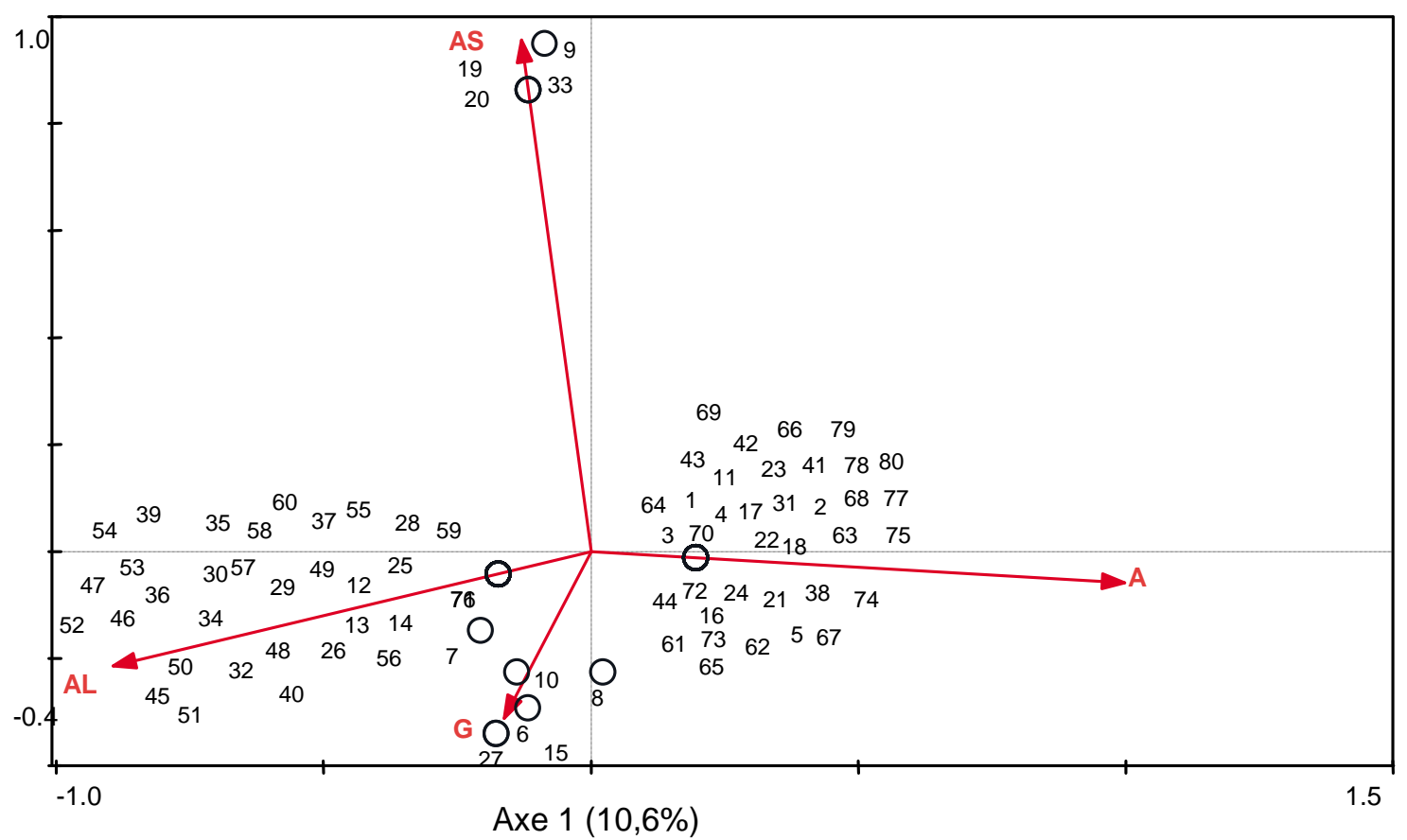

Figure 4: Diagramme de l'ordination des placeaux et des variables écologiques dans le plan factoriel des axes 1 et 2 de l'ACC (axes: 1 horizontal et 2 vertical).

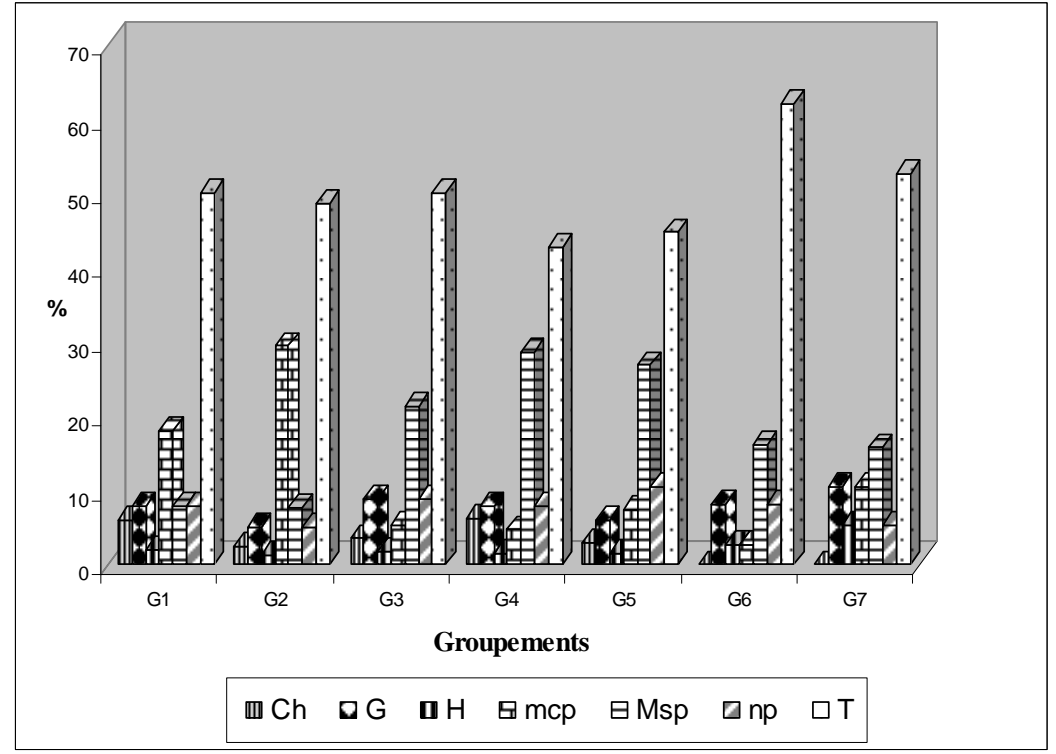

A) Spectre brut 


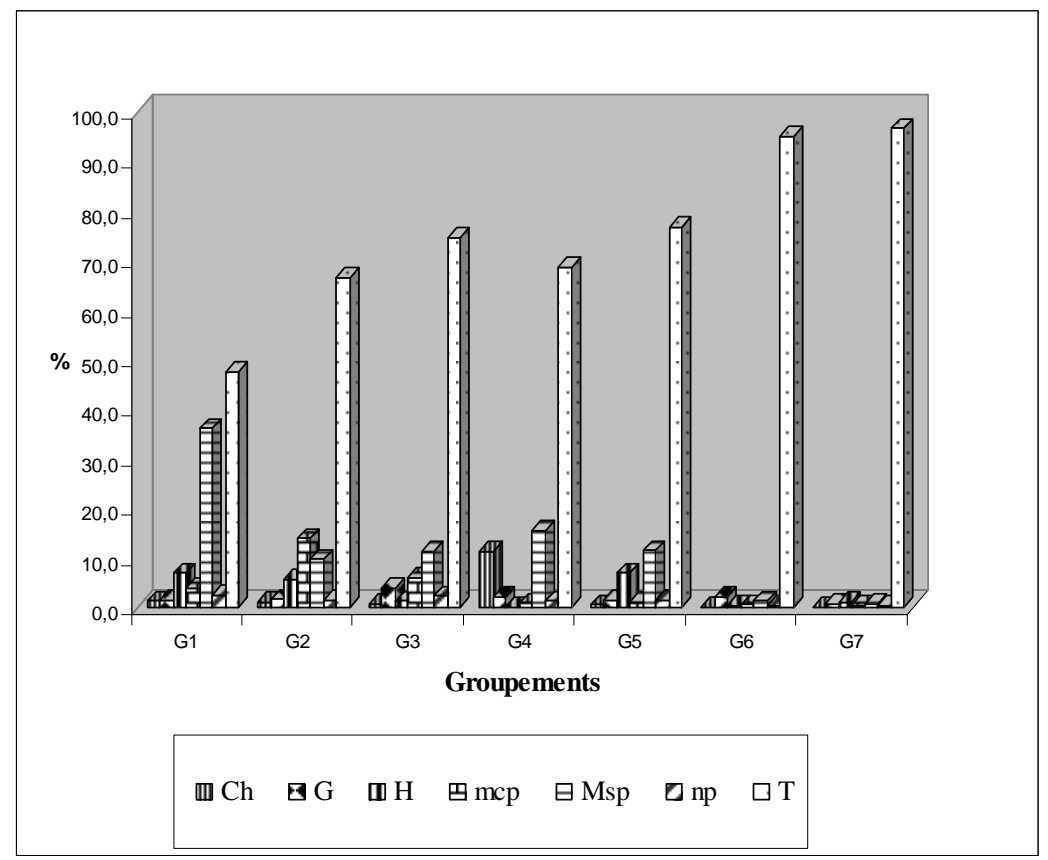

B) Spectre pondéré

Figure 5 : Spectres bruts (A) et pondérés (B) des formes biologiques des différents groupements.

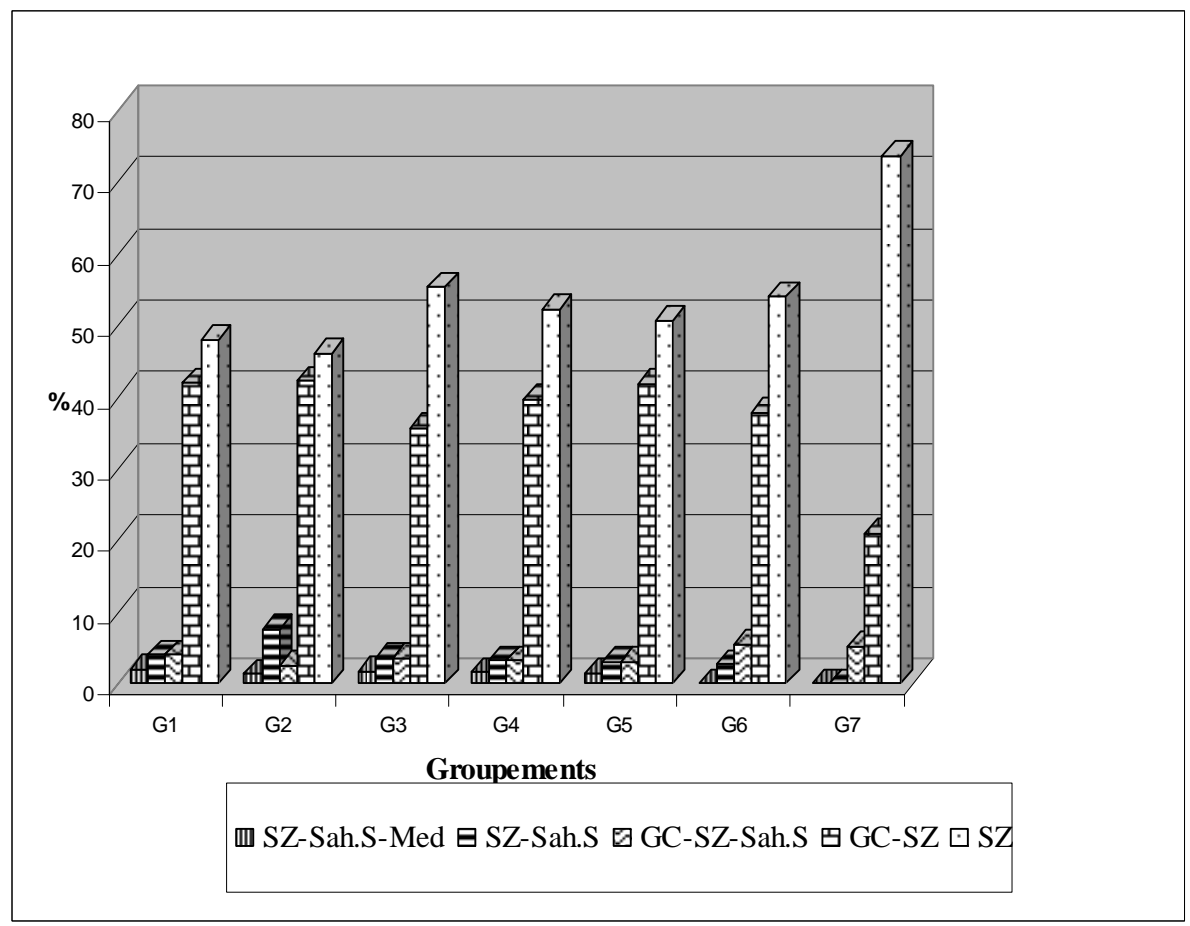

A) Spectre brut 


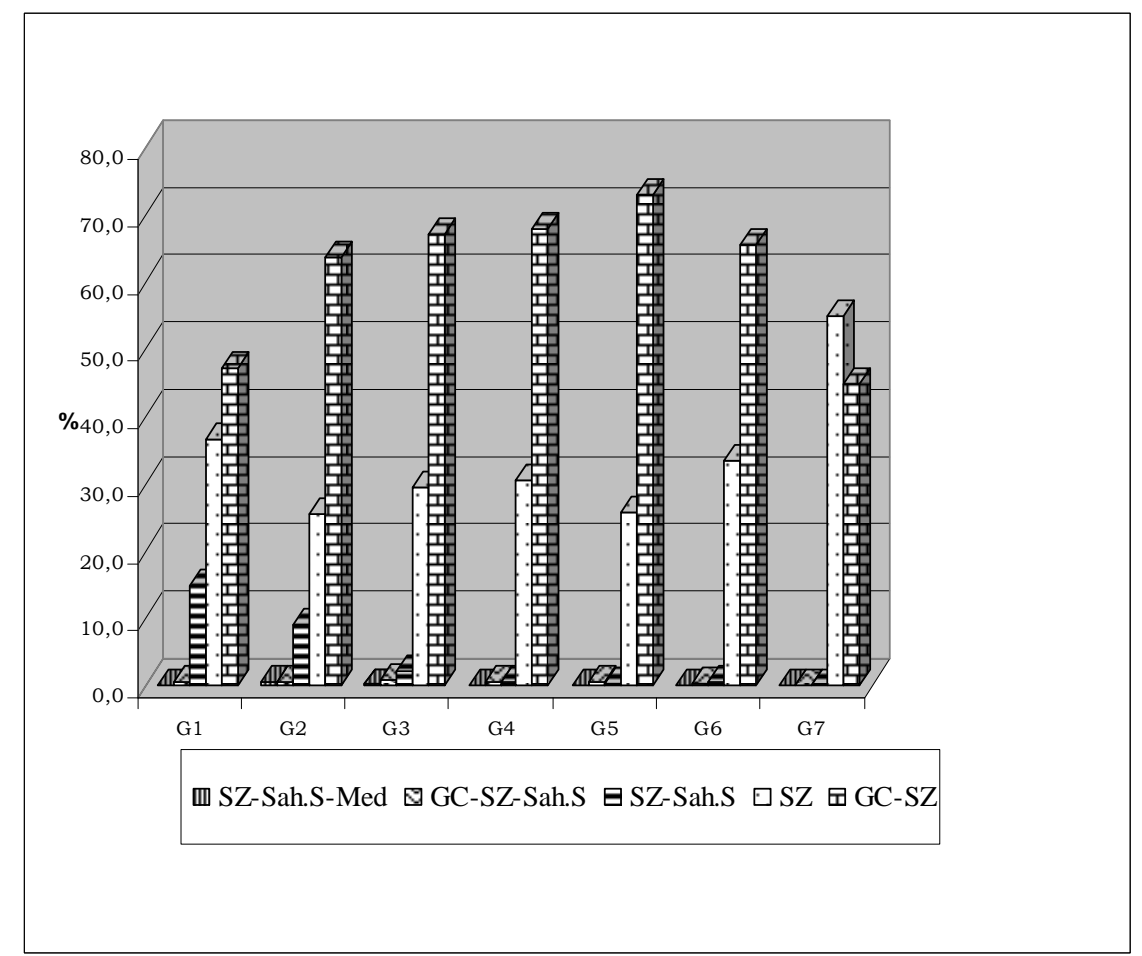

B) Spectre pondéré

Figure 6: Spectres brut (A) et pondéré (B) des types phytogéographiques des différents groupements.

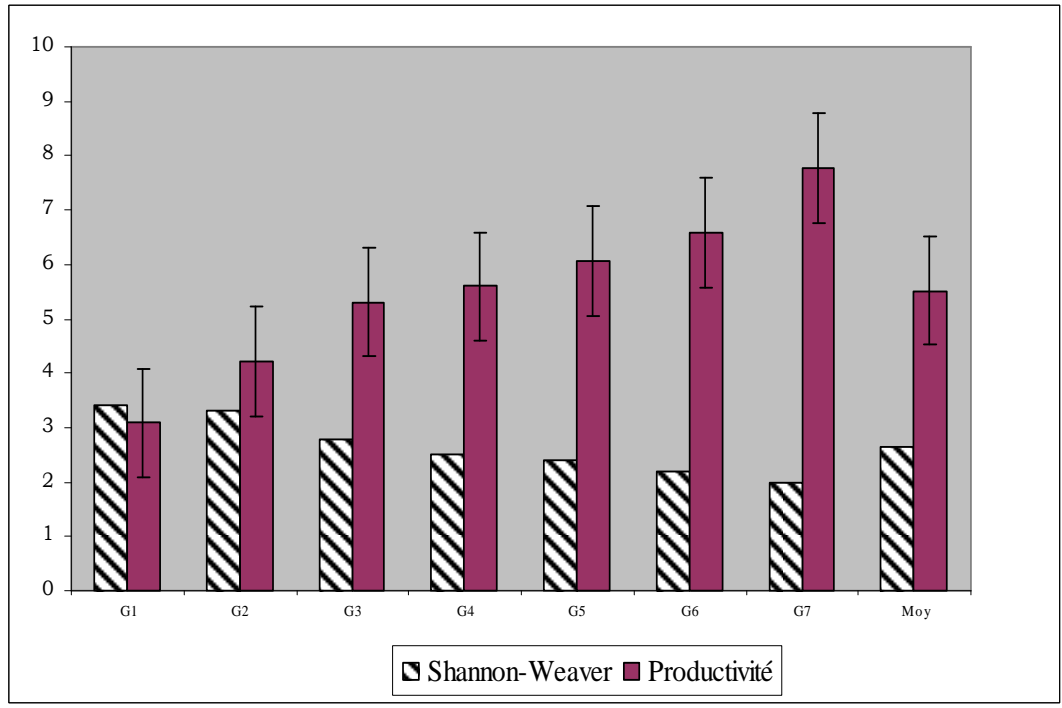

Figure 7: Rapport entre indice de Shannon-Waever et la Productivité au sein des groupements. 


\section{Conclusion}

$\mathrm{Au}$ terme de cette étude, 7 faciès de savane ont été identifiés sur le site dont quatre de savanes arbustives à arborées et trois de savanes herbeuses. Sur le plan floristique, 107 espèces ont été recensées parmi lesquelles 37 ligneuses et 70 herbacées, reparties dans 80 genres et 39 familles. Les différents groupements ont une richesse spécifique assez élevée variant entre 19 et 78 espèces. L'écologie des espèces montre la dominance des thérophytes qui est l'expression du caractère aride de la zone d'étude. Par rapport à l'affinité chorologique, ce sont les espèces soudano-zambésiennes et les guinéocongolaise-soudano-zambéziennes qui sont les mieux représentées. La présence considérable de ces espèces avec un nombre de GC-SZ qui augmente selon le groupement de la partie nord de la station vers sa partie sud, fait ressortir l'existence d'un gradient d'humidité et de profondeur du sol. Par ailleurs, le site est localisé dans la partie la plus arrosée du pays avec une pluviométrie moyenne de $700 \mathrm{~mm}$.

Tous les groupements identifiés sont diversifiés avec une productivité moyenne de 5,52 tonnes $/ \mathrm{ms} / \mathrm{ha}$. Celle-ci varie dans le sens inverse de la phytodiversité estimée à l'aide de l'indice de diversité alpha au niveau des groupements. Dans la mesure où l'utilisation des feux d'aménagement est inféodée aux pratiques des gestionnaires du parc pour la gestion de ces écosystèmes, il est nécessaire d'étudier l'impact des feux sur la dynamique de la végétation à dominance savanicole.

Ce travail a permis de caractériser la végétation du site étudié avant le traitement par le feu. Les indicateurs qui semblent être pertinents sur lesquels le suivi écologique doit porter sont : le nombre d'espèces ligneuses et herbacées, les types biologiques, la diversité alpha et bêta, la structure des ligneux ainsi que la productivité de la strate herbacée.

Les relevés doivent être répétés après l'application du feu afin d'appréhender les conséquences de ce dernier sur l'évolution de la composition floristique et de la structure de la végétation de la station explorée.

\section{REFERENCES}

Adam G, Jaeger P. 1977. Ecologie Suppression de la floraison consécutive à la suppression des feux dans les savanes et prairies de la Guinée (Afrique occidentale). C.R Acad. Sc. Paris, 282: $637-639$.

Amadou B. 2006. Analyse de la dynamique de l'occupation de l'espace et impacts sur les écosystèmes « étude de la croissance démographique dans la Réserve de Biosphère du $\mathrm{W}$ du Niger. Rap. UNESCO/MAB-UNEP/GEF.

Baina D. 2000. Contribution à l'étude floristique, écologique et phytosociologique de la forêt classée de Gourou Bassounga et des milieux cultivés adjacents. Thèse de Doctorat, Université Abdou Moumouni, Niamey, 151.

Barbier N. 2006. Auto-organisation et interactions spaciales des végétations semi-arides. Thèse de doctorat, Université Libre de Bruxelles, Bruxelles, 111p.

Casenave A, Valentin C. 1990. Les états de surface de la zone sahélienne : influence sur l'infiltration. (Collections Didactiques) ORSTOM : Paris ; 280 p.

Couteron P, Kokou K. 1994. Contribution à la connaissance de la végétation du Parc National de Taï, réalisation d'une clé de détermination sur des caractères végétatifs et analyse phyto-écologique d'un type de forêt. ENGREF/MAB-UNESCO, multigr., $48 \mathrm{p}$.

Dennis R, Meijaard E, Applegate G, Nasi R. Moore P. 2001. Impact of Human-Caused Fires on Biodiversity and Ecosystem Functioning, and their Causes in Tropical, Temperate and Boreal Forest Biomes. CDB Technical Series No. 5. Convention sur la diversité biologique: Montréal, Canada.

Diouf A. 2006. Etude des interactions biologiques au sein d'une végétation périodique du Niger par l'analyse des processus ponctuels, Mémoire de DEA, Université Libre Bruxelles, Bruxelles, 42p.

Diouf A. 2007. Dispositif de suivi des impacts des feux d'aménagement sur la végétation dans le Parc Régional W du Niger, Laboratoire GARBA Mounkaila UAM; Programme Régional Parc W, Composante du Niger, 12p.

Diouf A. 2012 Influence du régime des feux d'aménagement sur la structure ligneuse des savanes nord-soudaniennes dans le Parc du W (Sud Ouest NIGER). Thèse de 
Doctorat, Université Libre de Bruxelles, Bruxelles.

Diouf A, Barbier N, Lykke AM, Couteron P, Deblauwe V, Mahamane A. 2012 Relationships between fire history, edaphic factors and woody vegetation structure and composition in a semi-arid savanna landscape (Niger, West Africa). Applied Vegetation Science 15(4) : 488500.

Djitèye M. 1988. Composition, structure et production des communautés végétales sahéliennes : Application à la zone de Niono (Mali). Thèse de Doctorat, Université de Paris-Sud, Paris, 150 p.

Fournier A, Yoni M, Zombré P. 2000. Les jachères à Andropogon gayanus en savane soudanienne dans l'ouest du Burkina Faso : flore, structure, déterminants et fonction dans l'écosystème. Flore et Végétation de Burkina Faso et Pays Avoisinants, 3 ; 32p.

Guinochet. 1973. Phytosociologie. Collection d'Ecologie. Edition Masson et Cie ; 227p.

Houinato M, Sinsin B, Lejoly J. 2001. Impact des feux de brousse sur la dynamique des communautés végétales dans la forêt de Bassila (Bénin). Société Botanique de France, 148(3): 237-251.

Hutchinson J, Dalziel JM, Keay RWJ, Hepper FN, 1954-1972. Flora of West Tropical Africa (2 ${ }^{\text {nd }}$ edn, vol 1-3). The Whitefriars Press: London.

Inoussa MM. 2011. Dynamique des forêts claires dans le parc National du W du Niger Thèse de Doctorat, Université Abdou Moumouni, Niamey, 93p

Lamarque F. 2004. Les grands mammifères $\mathrm{du}$ complexe WAP. $U$ E, CIRAD, ECOPAS, $39 \mathrm{p}+51$ fiches.

Lebrun JP, Stock AL. 1991-1999. Enumération des Plantes à Fleurs d'Afrique Tropicale (vol 4). Editions des Conser. et Jard. Bot.: Genève.

Mahamane A. 2005. Etude floristique, phytosociologie et phytogéographique de la végétation du Parc Régional du W du Niger. Thèse de Doctorat, Université Libre de Bruxelles, Bruxelles, 516p.
Mbow C. 2009. Potentiel et dynamique des stocks de carbone des savanes soudaniennes et soudano-guinéennes du Sénégal. Thèse de Doctorat, Université Cheikh Anta Diop, Dakar.

Padonou EA, Assogbadjo AE, Bachmann Y, Sinsin B. 2013. How far bowalization affects phytodiversity, life forms and plant morphology in Sub-humidtropic in West Africa. African Journal of Ecology, 51: 255-262.

Peterson DW, Reich PB. 2001. Prescribed fire in oak savanna: fire frequency Effects on stand structure and dynamics. Ecological Applications, 11(3): 914-927.

Reich PB, Peterson DW, Wedin DA, Keith Wrage. 2001. Fire and vegetation effects on productivity and nitrogen Cycling across a forest-grassland continuum. Ecology, 82(6): 1703-1719.

Saadou M. 1990. La végétation des milieux drainés nigériens à l'Est du fleuve Niger. Thèse de Doctorat, Université de Niamey, Niamey, 395 p. + annexes.

Sankaran M, Hanan NP, Scholes RJ, Ratnam J, Augustine DJ, Cade BS, Gignoux J, Higgins SI, Le Roux X, Ludwig F. 2005. Determinants of woody cover in African savannas. Nature, 438(7069): 846-849.

Ter Braak CJF, Smilauer P. 1998. CANOCO Reference Manual and User's Guide to Canoco for Windows: Software for Canonical Community Ordination (version 4). Microcomputer Power: Ithaca, NY USA; $352 \mathrm{p}$.

Van Langevelde F, Van De Vijver CADM, Lalit Kumar, Van De Koppel J, De Ridder N, Van Andel J, Skidmore AK, Hearne JW, Stroosnijder L, Bond WJ, Prins HHT, Rietkerk M. 2003. Effects of fire and herbivory on the stability of Savanna ecosystems. Ecology, 84(2): 337-350.

Villecourt P, Schmidt W, Cesar J. 1980. Pertes d'un écosystème à l' occasion du feu de brousse (Savane tropicale de Lamto, Cote d'Ivoire). Revue d'Ecologie et de Biologie du Sol, 17: 7-12. 